\title{
Neuroimaging Helps to \\ Clarify Brain Affective Processing \\ Without Necessarily Clarifying Emotions
}

\author{
Peter Walla and Jaak Panksepp
}

Additional information is available at the end of the chapter

http://dx.doi.org/10.5772/51761

\section{Introduction}

Literally, Neuroimaging gives us deeper insight into the brain's function. But, what actually is its function? The brain is an organ, almost just as our lungs and hearts. The big difference is that we can see the imprints of evolutionary progressions in its organization. Although the stamp of evolution is clearly imprinted in the genes that govern the functions of all the other organs, that stamp is special within the brain: Brain functions that evolved earlier are concentrated in more caudal and medial regions of the brain while those that emerged later (e.g., neocortex) are concentrated more rostrally and laterally. Thus, although our brain is an organ like no other in the body, we should envision the mind as largely the product of this bodily organ, although mind is surely not disconnected from other bodily functions.

Our heart pumps blood to provide all body parts with essential chemicals. The blood pumping is the function (or the mechanism) and the delivery of chemicals the goal. Likewise, the brain has various intrinsic functions and evolutionary goals (the "what for" evolutionary issue, in Dan Dennett's terms, as contrasted to the scientifically more workable "how come" dimensions of brain mechanisms). The brain's overall function is to process external information, as referenced to internal survival issues that are often coded as affective states, and the goal is to produce behaviours that sustain existence. Thinking of Darwin's (1859) idea that fitter organisms are better survivors the brain must have evolved to adapt the behaviour of its carrier organism to an ever-changing environment, however the adaptive behaviours also need to be referenced to internal survival issues. Sensory input representing the outer world is processed and forms an important basis for decisions to be made, which need to be related to internal survival issues, which in turn form the basis for actual behaviour in terms of movement (any form of muscle contraction). Sensory input, information processing and motor output, these are the well-accepted major steps of the 
brain's function (see Walla, 2011). However, the internal "set-points" - the within brain value referencing of behaviour - is often left out of the equation. Here we also seek to bring that dimension, as reflected in the affective lives of organisms (Panksepp, 1998; Panksepp \& Biven, 2012), back into the discussion more explicitly, in scientifically testable ways.

This chapter focuses specifically on the affective side of information processing, especially taking into account findings from neuroimaging studies. In addition, the issue is raised that the term "emotion" is at times confusingly and interchangeably used between and even within disciplines that study the brain and mind, which is itself delaying advances in our understanding of the brain. Without conceptual agreement on key terms such as affect, emotion and motivation, along with the recognition that each has undergone multi-tiered evolutionary progressions, the likelihood of talking past each other increases, as with the perennial debates between basic emotion theory and constructivist approaches to similar problems (e.g., Lindquist, et al., 2012; Zachar \& Ellis, 2012).

Despite the rather confusing existing terminologies, it must be admitted that neuroimaging technologies have shed considerable light on functions underlying emotions. These mainly include cognitively preconscious and unconscious aspects (Berlin, 2011), which are not easily accessible by questionnaire-based investigations (although they may be mentalized with depth-psychoanalytic interviews (see Kaplan-Solms \& Solms, 2000), giving neuroimaging technologies one of their most attractive feature. The brain knows (and maybe experiences) more than it admits at the cognitive tertiary-process level (Solms \& Panksepp, 2012), and among the objective tools, only neuroimaging (and startle reflex modulation; see Walla et al. 2011) has access to this knowledge. In fact, the advent of sophisticated brain imaging methods has facilitated demonstration of the existence of various apparently non-conscious brain processes that guide human behaviour within both affective and the cognitive domains, even as certain aspects like affective changes often reflect brain changes that are not easily measured with fMRI (functional Magnet Resonance Imaging) approaches which require precise timing, but can be dramatically envisioned with PET (Positron Emission Tomography) scans that are not subject to rigid temporal constrains (Damasio, et al., 2000). In any event, there is more to emotion than subjective feelings, with many unconscious underpinnings, and it is important to better understand all levels. For many issues we simply need to look inside the box that processes all the information, for only a fraction of this turns into responses that can be measured via self report. Also, since it is well-established that cognitive and affective processes are often in a see-saw balance (Liotti \& Panksepp, 2004), in fMRI environments that use discrete emotional-cognitive stimuli (e.g., facially expressive pictures), it is often best to instruct subjects to experience their feelings in the scanner without reporting on their internals states, and then to harvest subjective reports after the brain-imaging session is completed; through such procedures, it is clear that affective changes are typically directly related more to subcortical arousals, and inversely correlated with cortical ones (Northoff, et al., 2009).

Our goal here is to share relevant research to highlight some of the points already made. Besides introducing some of the key findings of various neuroimaging methods and proposing a clear and distinct use of the terms "emotion" and "affective processing", we 
also entertain clear ways to separate cognitions from affective processing and emotion (although a lot of interaction occurs). Mauss and Robinson (2009) finished their abstract by concluding that experiential, physiological, and behavioural measures are all relevant to understanding emotion and that they cannot be assumed to be interchangeable. We agree and add that a full-blown emotion is the result of physiological activity-both of brain and body--which we call affective processing, but that affective processing alone does not yet qualify for an emotion. In animals, emotions are evident in distinct behavioural displays, along with complex changes, in which all relevant components are already organized at subcortical levels, along with some form of primal affective states as monitored by the rewarding and punishing properties of those circuits (Panksepp, 1998). However, those low levels of the brain do not elaborate the many cognitive processes that always accompany emotional arousal in humans. The distinction between affective processing and emotion shall be the most important basis of the proposed emotion model (see final section of paper). Here affect is seen as the raw valenced aspect of emotional arousals, while the concept of emotion remains more indeterminated. Although it has traditionally included everything, from diverse cognitive, behavioural and autonomic associates to very subtle and diverse feeling states, we suspect that it is time to re-simplify this term, so it does not cause as much mischief as it has, ever since Darwin brought it to the scientific table.

\section{Further background}

The idea that the primary purpose of the brain is to produce some sort of movement fits the notion of the evolutionary process called cephalisation, the creation of a separate specialised head enclosing a central brain. As organisms developed limbs for locomotion, their body shape also began to change. The body took on a longitudinal forward-facing position and the sensory organs began to move to the front and/or develop at the front of the body, where first contact with new environmental stimuli would be made. Since a major attribute of the brain's overall functioning is to process sensory input, it seems obvious that having sensory organs at the front of the body and the brain close by is a big advantage. Thus, by simply observing the stages of evolution it can be said that the movements allowed our brain to produce complex action patterns that reflect various within-brain processes, including both unconscious and phenomenally experienced ones. It is always hard to imagine such major evolutionary steps, but doing so tremendously facilitates our understanding of how the brain works. It also affords discourse sufficient complexity to reduce the likelihood that investigators working at different evolutionary levels indulge in seemingly substantive but often empty controversies.

An example may help here: In trying to understand both unconscious and phenomenally experienced action tendencies, it is critical to recognize that an ancestral urge to move forward and approach resources-e.g., a reward SEEKING system-has both unconscious and experienced brain functions. This urge was built into the brain early in brain evolution (i.e., it is a primary-process) shared in basic form - as "a major brain reward system"--by all vertebrates; it is experienced as a diffuse positive enthusiasm to pursue resources (the felt component is inferred from the rewarding property of this system, even in the absence of 
higher brain functions as in decorticate animals), but it is one that has remained linked to the emergence and refinement of sophisticated action routines permitted by cephalization and hence ever more sophisticated cognitive capacities to guide behaviour (Panksepp, 1998; Panksepp \& Moskal, 2008). It looks like a series of such fundamental affective systems govern the basic "secondary process" learning and memory functions of the brain that, to the best of our knowledge, operate totally unconsciously (affective memory). The harvesting of memories about the world (semantic and episodic memory) provides opportunities for the highest regions of the brain, whose functions are best visualized with modern brain imaging - from fMRI to MEG (Magnetoencephalography) procedures - to generate diverse higher mental functions, many of which reflect subconscious processing while some appear as experienced thoughts within our minds (conscious processing).

Now, let's take a closer look at our brain's function. We will especially focus on the processing of external information entering the brain via sensory organs. Put simply, there are two major aspects of any sensory input that update our brain with the conditions of the world outside in order to adapt behavior. One aspect refers to detailed semantic information, i.e. "What is it that I see through my eyes, hear through my ears, or detect with any of my other sensory systems?" That aspect reflects the rationale or cognitive domain. The other aspect is not detailed at all and refers to rather abstract information, i.e. "Is what I see through my eyes, hear through my ears, or detect through any of my other sensory systems supporting my life or is it detrimental?" That aspect reflects the affective domain, which is pre-cognitive in the sense that internal neuro-affective emotional states intrinsically guide action tendencies since they arise from the neural circuitries of instinctual emotional action tendencies. By the way, the sense of olfaction might be the modality that first delivered the mammalian brain (or maybe even the brains of all vertebrates) with external information leading to strong affective processing (see Walla , 2008; Walla \& Deecke, 2010). In general, affective state processing appears to be more primitive than cognitive information processing and might have evolved as a first "evaluation system" guiding behavior on the basis of more precise external information processing, slightly above the unconscious behaviors that reflect reflexes and other completely automatic processes (e.g., cardiac dynamics) that proceed without any obligatory mental contents.

However, we do not wish to suggest that automatic processes are always unconscious since at the foundational level we have "instinctual" systems that are more than reflexive in that there are large-scale systems that guide dynamic instinctual behavioral routines that already evaluate the environment for conditions that support or detract from survival. They may exist without any sophisticated cognitive mind, but may already have developed the capacity for affective experiential capacities as a primal way to steer behavior and guide learning based on incoming sensory inputs-such arousals feel good and bad in various ways, and promote basic learning mechanisms (e.g., operant conditioning; affective memory). If certain life events lead to bad sensory feelings such as pain, some of these systems promote various escape and avoidance behaviors that reduce those feelings (by withdrawal from negative affects). If life events lead to good feelings, there are intrinsic systems that are well positioned to increase those feelings (via various approach behaviors). 
These low-level instinctual-integrative systems are the ones we call intrinsic primaryprocesses. Thus, we already have distinct sensory-affects and emotional affects at the very foundations of the mind and brain (Panksepp \& Biven, 2012; Solms \& Panksepp, 2012). Further, both of these types of primal affects are modulated by bodily state-e.g., hunger and thirst. The way we can determine such affective states of mind in animals is by direct evaluation of the rewarding and punishing properties of artificial arousal of emotional action systems as with direct electrical deep brain stimulation (DBS) (Panksepp, 1998, 2005).

The topmost, tertiary-process, levels of our brain's function can thus be extended to conjoint affective and cognitive processing. Panksepp's Affective Neuroscience (1998) framework states that the affective aspects of a stimulus are processed before and independently of any cognitive aspects, given their more ancient role in decision-making. This makes sense given that key structures engaged in the generation of primal emotions are found to be mainly subcortical (see later)-caudo-rostrally areas such as periaqueductal gray, hypothalamus, ventral striatum, bed nucleus of the stria terminalis, dorsomedial thalamus, septum and amygdala, as well as at higher levels in regions such as cingulate cortex, insula, and orbitofrontal cortex. Although some have posited that the amygdala lies at the heart of affective life (LeDoux, 1996), while others have bestowed a primary role on the insula (Craig, 2009), it is quite clear that humans with both of these brain regions completely burned out by brain diseases have vibrantly rich affective lives (for most compelling case study and relevant literature, see Damasio, et al., 2012).

In contrast, most explicit cognitive processing is heavily neo-cortical. Additionally, subcortical structures are evolutionarily older than cortical structures, meaning that the first elements of decision-making must have involved basic affective processing. Thus, older and faster are two distinctive features of affective processing versus cognitive processing. This has important consequences for the relationship between locomotion and cephalization. Moving forward means to continuously face different environments and it is of utmost importance to evaluate all new stimuli with respect to their survival value. Much of the psychomotor energy for this persistent urge of the nervous system is due to arousal of the mesolimbic SEEKING system (Ikemoto \& Panksepp, 1999; Alcaro, et al., 2007, 2011; Panksepp \& Moskal, 2008). At the primary process level (see Panksepp, 2011), it is more important to evaluate the survival value of external stimuli as well as internal states than to know what they actually are, giving affective information processing a more primary survival role than cognitive information processing. Further, affective processing needs to be sub-categorized into at least three levels of analysis: sensory affects (such as taste and olfaction), homeostatic affects (such as hunger and thirst mediated by bodily interoceptors), and emotional affects (the most subtle, and most important for psychiatric issues, which seem to arise from the intrinsic affective action schema of the brain).

\section{Subcortical structures process non-conscious affective information}

Most neuroimaging studies find that various subcortical and some cortical structures are involved in affective information processing. According to Tracy and Randles (2011), four key 
investigators in emotion research agree that "basic emotions" arise from activities in subcortical structures. In particular, Panksepp and colleagues (see Panksepp \& Biven, 2012; Panksepp \& Watt, 2011) assert that only subcortical structures house genetically determined, fixed emotional networks whose activities generate various rewarding and punishing states, while neocortex provides the necessary cognitive space to think about them. While all four scholars discussed by Tracy and Randles come up with slightly different sets of basic emotions, indicating there are still abundant ambiguities in the field, only one of the above is based on rigorous neuroscientific analysis of the underlying brain systems with DBS (e.g. Panksepp, 1992). In any event, all agree that emotions start with some kind of subcortical processing.

By far the most frequently noted subcortical structure is the well-known amygdala (one in each hemisphere). This structure is the size of a hazelnut and it has become the star in affective processing (to cite just a few key investigators: Halgren et al., 1978; LeDoux, 1993; Bechara et al., 1995; Adolphs et al., 1995; Calder et al., 1996). Many others also report that this structure is critically involved in affective information processing, although it does not seem to be essential for experienced affective feelings (Damasio, et al., 2012). While it seems obvious that increased activity in the amygdala is important for the learning of fear, it is wrong to assume that amygdala activity alone is enough for generating feelings of fear. In fact, it is more involved in the learned information processing related to fearful arousals, and much of that secondary-processing can be unconscious. In fact, it has been shown that amygdala activity as a consequence of visual negatively valenced stimulation occurs and changes behaviour even in the absence of conscious visual perception (e.g. Öhman, 2002). Morris et al. (1999) nicely demonstrated in an fMRI study that amygdala activity is also increased in case of unseen negative emotional information content (not consciously perceived visual stimuli). Another team of investigators titled their paper "Affective blindsight" (Hamm et al., 2003). They found startle reflex enhancement as a consequence of visual fear conditioning in a cortically blind patient. Their data suggest that visual subcortical pathways including the amygdala are sufficient to activate negatively valenced affective information processing in humans in the total absence of visual awareness.

From the idea that subcortical structures are the ones that process basic emotion-related information it is not far to the idea that affective processing happens outside of cognitive awareness. Some investigators have elaborate ideas about "unconscious emotion". Zajonc (2000) addressed the issue of non-conscious (or unconscious) emotions. For instance, in a book titled "The Nature of Emotion: Fundamental Questions" (edited by Ekman and Davison in 1994) he wrote an essay with the title "Evidence for non-conscious emotion". In 2003, Berridge and Winkielman report about evidence supporting the idea of genuinely unconscious emotion (particularly unconscious liking). The authors report about their findings that despite the absence of detectable subjective shifts in affective experiences, subliminally induced unconscious liking can influence concurrent consumption of a sweetened drink. After all that, we also believe that much unconscious processing occurs related to "emotion", but what's unconscious here should be labeled "affective information processing" and not "emotion", because emotion is not information processing, it is the output of it as we propose (see final section of this paper). 
In 2000, Zajonc also proposed in an article provocatively entitled "Closing the debate over the independence of affect" that the separation of affective and cognitive neuroscience exists and makes sense. This gives Panksepp's "Affective Neuroscience" (1998) a stand-alone character and it might also support the notion to exclude any intrinsic cognitive aspects in an emotion definition. Cognition is a separate, parallel function, which of course can and does synergize with as well as interfere with affective information processing, but it adds little to see cognition as an intrinsic aspect of primary-process emotionality, while at a tertiary-process level, it clearly has a role. In any event, affective information processing can and certainly does influence cognition. Cognitive influences on affective processing are top-down, whereas affective influences on cognition are rather bottom-up. In some neuroimaging studies about "emotion" neural structures that have been shown to be involved in some sort of cognition have commonly been found to be active (Lindquist, et al., 2012). Unfortunately, such findings have been commonly interpreted as "cognitive aspects of emotion" it may be wiser to see them as distinct associated processes. It may well be that at some point in information processing, perhaps shortly before a decision is made, affective information merges with cognitive information. In this combination, both initially separate processes subserve decision-making. However, a clear distinction should be made and a clear line drawn.

\section{Emotion through facial expression}

Much of the human basic-emotion literature has focused on emotions elicited through facial expressions (e.g. Ekman et al., 1987; Izard, 1971; Tracy \& Robins, 2008). There is considerable evidence showing that, for instance, a facial expression of disgust elicits brain activity in the observer's brains other than that evoked by a disgusting non-facial picture (Faces versus scenes; e.g. Sabatinelli et al., 2011). Unpublished electroencephalography (EEG) data of a diploma student supervised by Peter Walla show that pictures of natural scenes of different emotional categories elicit larger deviating event-related potentials (ERPs) between emotion categories than facial expressions of the same emotion categories. This is the case for early (below $200 \mathrm{~ms}$ ) and for later (200 ms to $800 \mathrm{~ms}$ ) time windows.

Narumoto et al. (2001) wrote a paper entitled "Attention to emotion modulates fMRI activity in the right superior temporal sulcus (STS). However, it is clear that they scanned their study participants while exposing them to facially expressive stimuli. Their finding was that the right STS, a structure that they found involved in face processing alone turned out to be more active in case of emotion attention. The point here is that a facial expression is an emotion of the person the face belongs to. The image of a facial expression is not necessarily in itself a matching affective stimulus such as the scene that elicited affect in the person demonstrating the facial expression. Again, imagine a disgusting scene versus a disgusted face. Let us call the difference "direct versus indirect affective information" (or first- and second-hand affective information). It instantly becomes clear that indirect affective information as communicated via facial expression can be misinterpreted and actually lead to different affective processing and a different emotion in the observer of a facial expression. Second-hand affective information is influenced (or biased) by all sorts of context-dependent information. For example, an angry facial expression can be the result of 
true primary-process anger, but it can equally likely be the result of tertiary-process victory ("DAMN IT, I WON!", see figure 1) and would thus be the result of positive affective information. It seems that context plays a crucial role in the higher-order perception of emotion as in human facial expressions, and much of the existing literature has never attempted to ferret out levels of control within the brain or mind that may reflect synergistic or distinct affective processes. That failure could have led to enormous and persistent controversies in human emotion research, especially when it comes to perennial debates between advocates of basic emotion theory, e.g., Paul Ekman and Cal Izard, and those who pursue constructivist agendas such as Lisa Feldman Barrett and Jim Russell (for a thorough discussion, see Lindquist, et al., 2012, and associated commentaries). On the other hand, in scenes context is always present eliciting affective processing and emotions more directly.
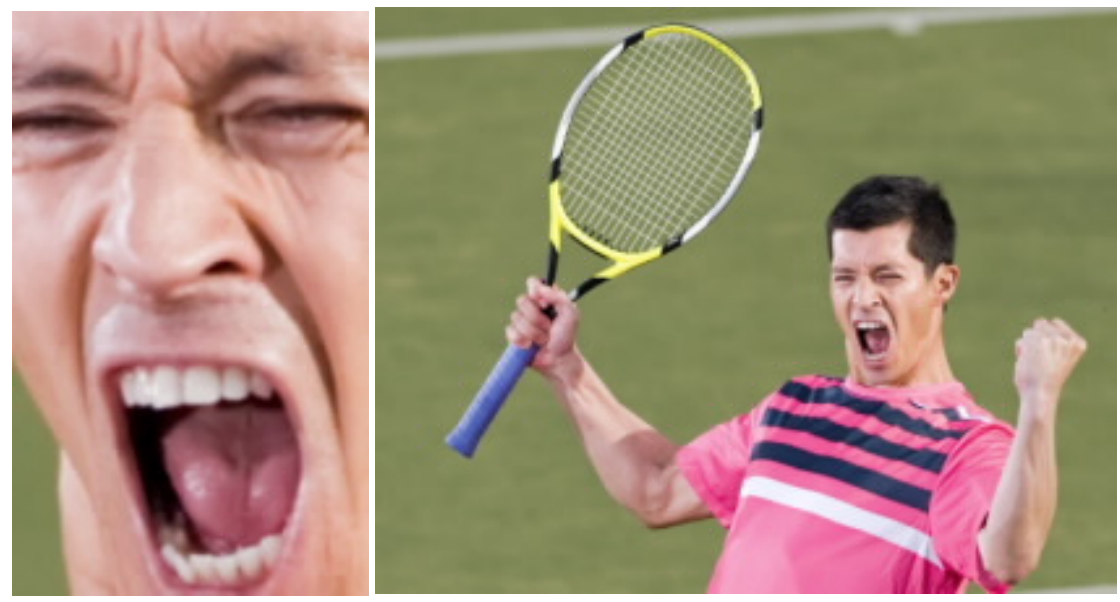

Figure 1. Left: facial expression alone looks like "anger" or "pain". Right: with context it turns into a quite positive and rewarding emotion communication (Game - Set - Match). iStockphoto.

\section{Affective processing and self-reference}

Self-reference might be critically involved in some specific emotion-related brain processes (Northoff et al., 2006; Garrett and Maddoc, 2006). The insular cortex has been suggested to be involved in processing self-referential information in a magnetoencephalography (MEG) (Walla et al., 2007) and an electroencephalography (EEG) study (Walla et al., 2008). In these studies, pairs of possessive pronouns and nouns (e.g. my pencil; his pencil) were used to provide controlled independent variables reflecting varying conditions of ownership. These were compared with a neutral condition (e.g. a pencil) with no personal reference. Two temporal time windows were found to demonstrate interesting effects. In an early time window, the investigators found brain activity elicited by both referenced conditions "my pencil" and "his pencil" to differ from the unreferenced, neutral condition (a pencil). Figure 2 shows MEG data reflecting this finding. In a later time window though, "my pencil" elicited different brain activity compared to "his pencil", a clear sign of self-referential 
information processing (Figure 3). Figure 4 provides localisation results leading to the insular cortex hypothesis.

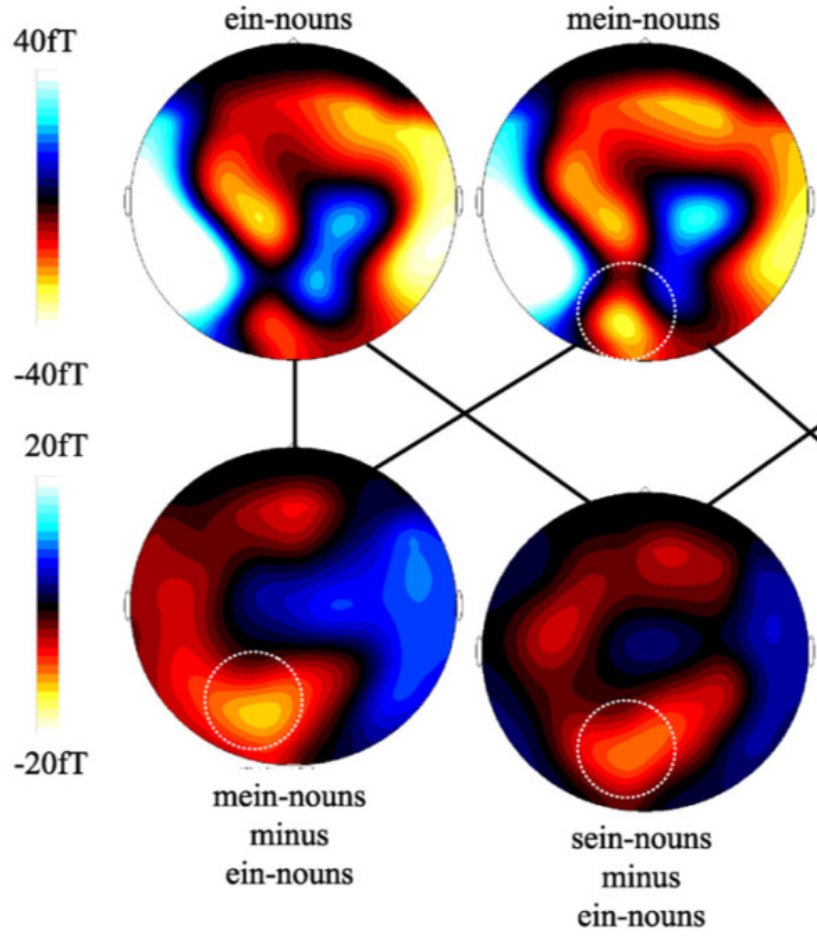

Distribution of significant difference activity: raw data
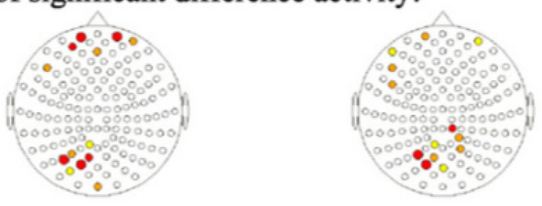

Distribution of significant difference activity: normalised data
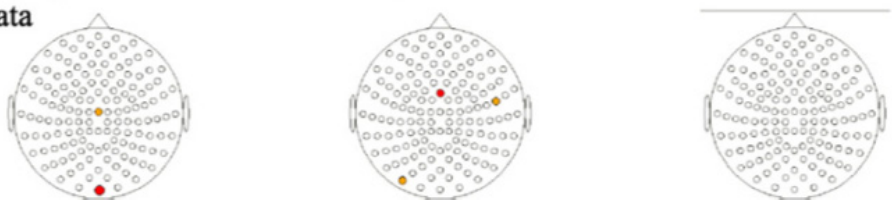

Figure 2. MEG maps. Early neurophysiological effect: MEG maps (magnetic field distributions) averaged across depth of word processing and across all study participants for the time interval from 200 to $300 \mathrm{~ms}$ after stimulus onset. First line: one map for each of the three conditions of pronoun ("ein" ("a"), "mein" ("my"), "sein" ("his")). Second line: difference magnetic field distributions related to comparisons (subtractions) between each possible pair of pronoun condition ("mein" vs. "ein", "sein" vs. "ein", "sein" vs. "mein"). Sensor areas where t-tests resulted in significant differences are marked 
with a white dotted circle. Third line: t-maps showing the distribution of significant differences for each of the above-mentioned comparisons (raw data). Note that "mein" vs. "ein" and "sein" vs. "ein" both resulted in significant differences, whereas no differences occurred for the comparison "sein" vs. "mein". Fourth line: t-maps showing the distribution of significant differences for each of the abovementioned comparisons (amplitude-normalized data). Note that hardly any differences occurred (adapted from Walla et al., 2007 with permission).

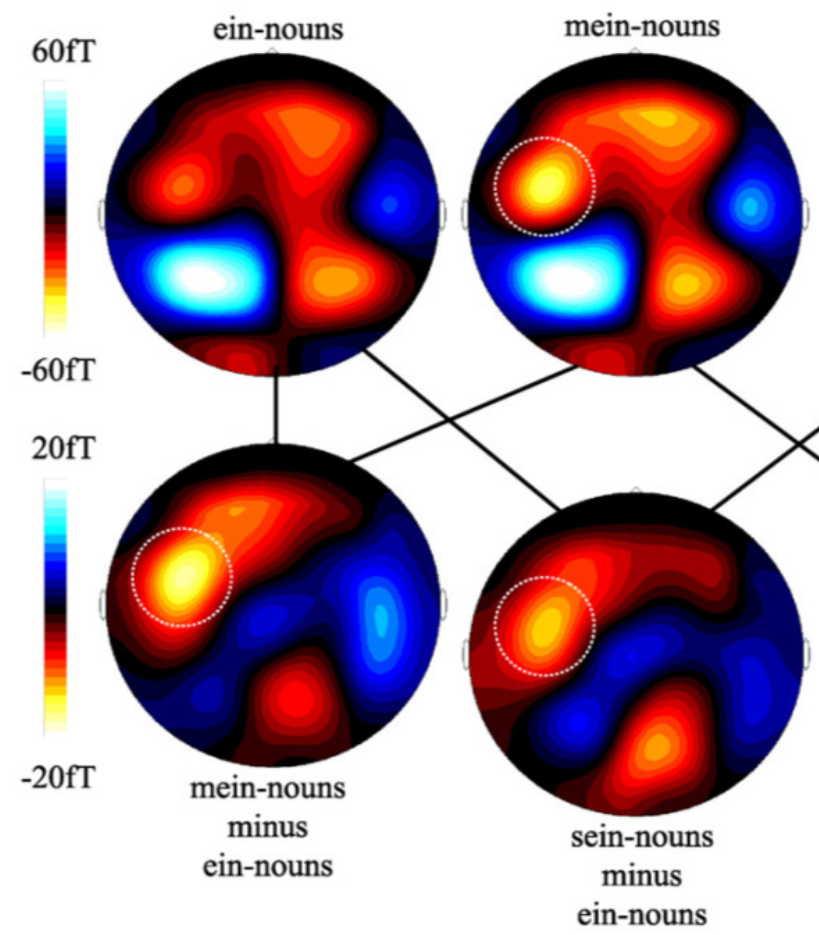

Distribution of significant difference activity: raw data
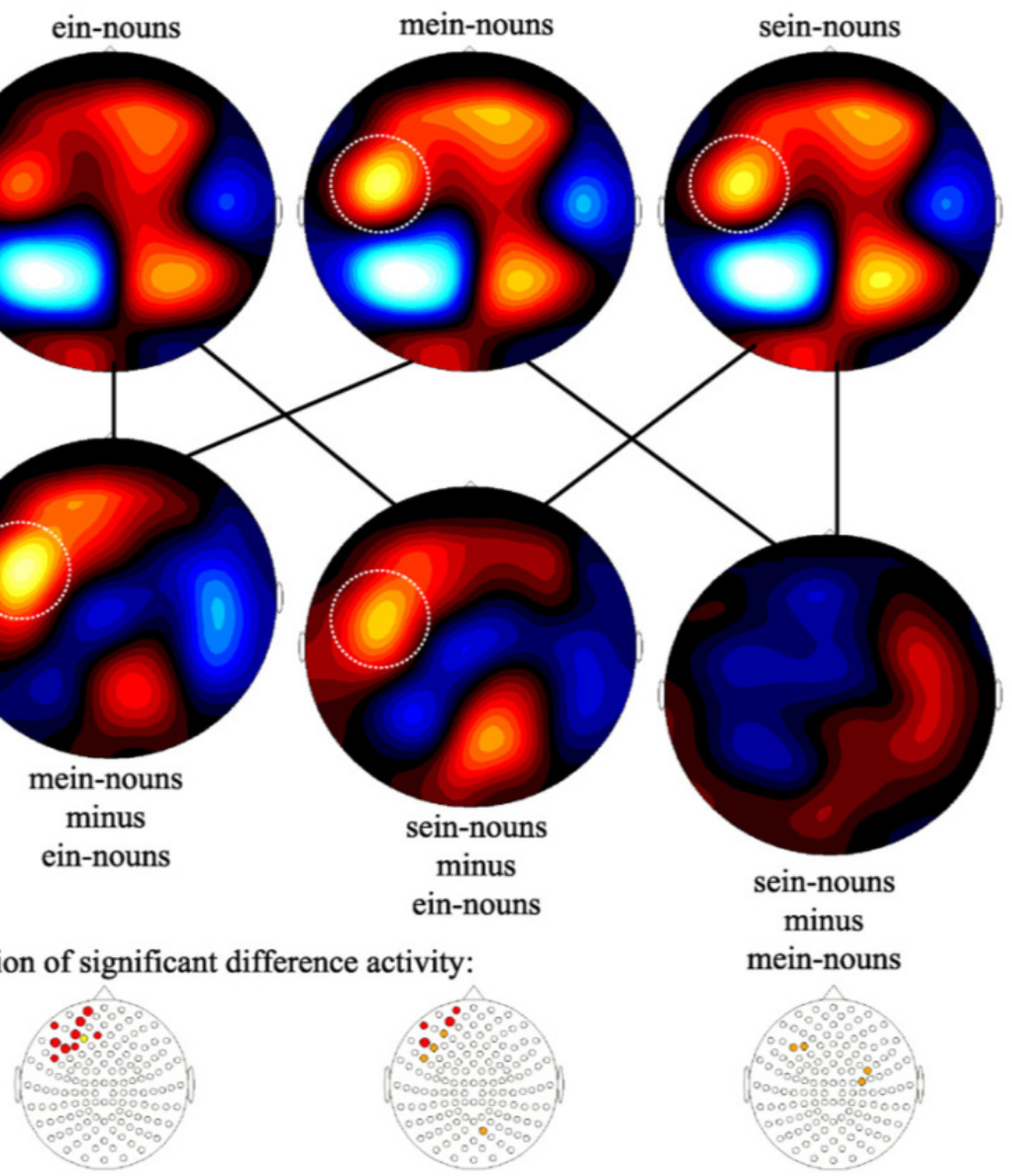

\section{Distribution of significant difference activity:}

\section{normalised data}

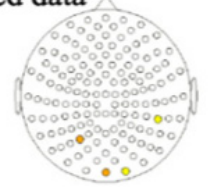

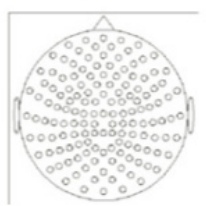
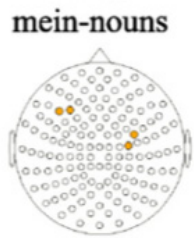

Figure 3. MEG maps. Later neurophysiological effect: MEG maps (magnetic field distributions) averaged across depth of word processing and across all study participants for the time interval from 
500 to $800 \mathrm{~ms}$ after stimulus onset. First line: one map for each of the three conditions of pronoun ("ein" ("a"), "mein" ("my"), "sein" ("his")). Second line: difference magnetic field distributions related to comparisons (subtractions) between each possible pair of pronoun condition ("mein" vs. "ein", "sein" vs. "ein", "sein" vs. "mein"). Sensor areas where t-tests resulted in significant differences are marked with a white dotted circle. Third line: t-maps showing the distribution of significant differences for each of the above-mentioned comparisons (raw data). Note that "mein" vs. "ein" and "sein" vs. "ein" both resulted in significant differences. In addition, the comparison between "sein" and "mein" also resulted in significant differences at some of the sensor sites (no such differences were found during the early period of time). Fourth line: $t$-maps showing the distribution of significant differences for each of the above-mentioned comparisons (normalized data) (adapted from Walla et al., 2007 with permission).
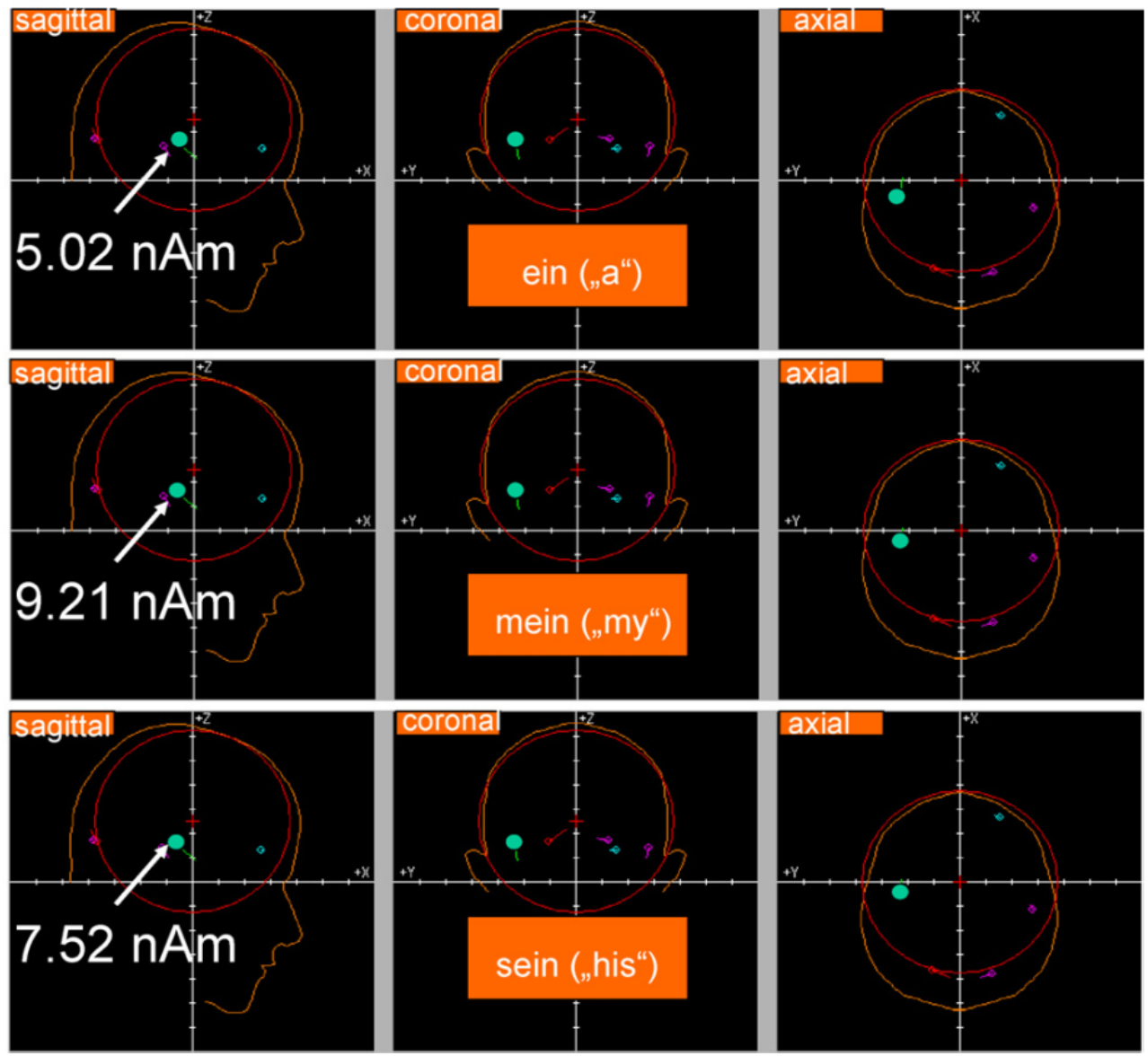

Figure 4. MEG source localisation results. Source localization for the later time range (500-800 ms): for the averaged data set (across 22 participants) the magnetic field distributions related to all three kinds of pronoun are displayed for a particular time point within the time window from 500 to $800 \mathrm{~ms}$, which was found to include significant differences over left temporal and left fronto-temporal sensors depending on the kind of pronoun. The localized dipole in this respective region shows stronger brain activity as reflected by dipole strength ( $\mathrm{nA} \mathrm{m}$ ) related to both personal pronouns compared to the 
neutral pronoun (strongest activity in the "mein"("my") condition). The location of this dipole is interpreted as left temporal. Most likely it is the left insular cortex that is able to discriminate between self and other (adapted from Walla et al., 2007 with permission).

The above-mentioned EEG study by Walla et al. (2008) revealed very supportive similar findings. Together with the MEG findings the "multiple aspect theory" of self-awareness was created and the insular cortex was suggested to be critical for self-reference processing. In an independent EEG study by Herbert et al. (2011a), confirmative date were collected with respect to a self-other distinction (Figure 5), and these self-reference findings were even extended to the emotion domain. From $350 \mathrm{~ms}$ onwards, processing of self-related unpleasant words (e.g. my fear) elicited larger frontal negativity compared to unpleasant words that were related to the other (e.g. his fear) or that had no reference at all (e.g. the fear).

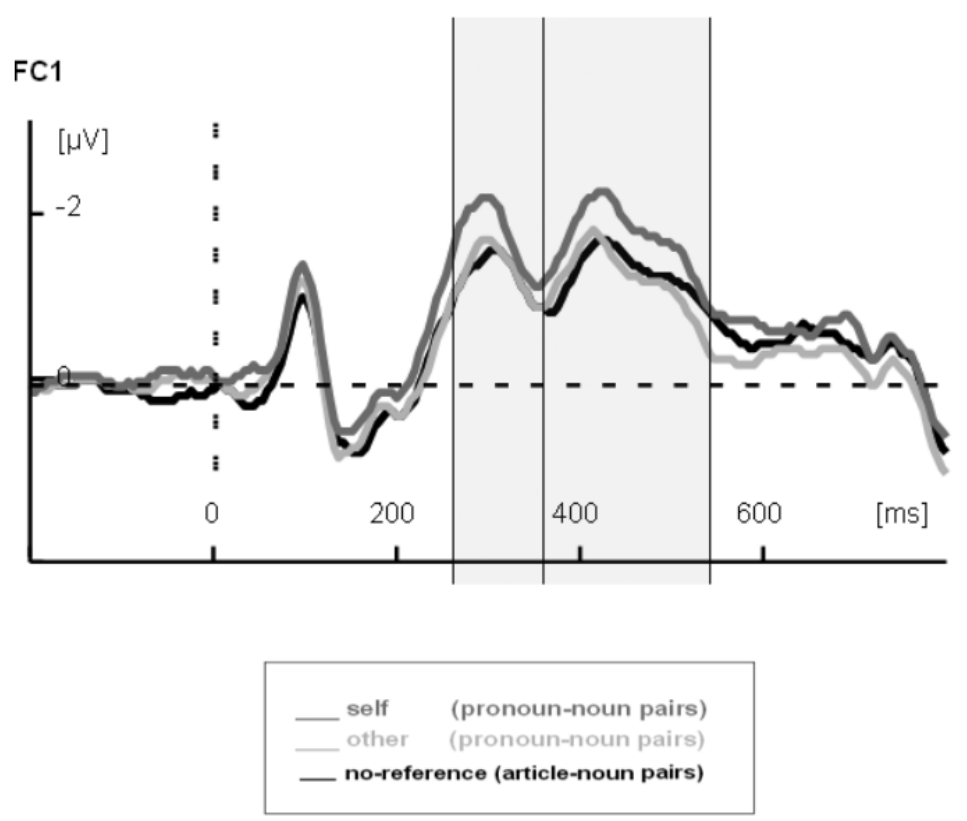

Figure 5. Main effects of self-other reference. Grand average ERP waveforms at posterior and anterior electrodes during reading of self- and other-related pronoun-noun pairs and non-referential articlenoun pairs (adapted from Herbert et al., 2011a with author permission).

The results of a recent fMRI study by Herbert et al. (2011b) revealed the neural structures that mediate self-reference in emotional conditions. The study demonstrates that the activity level of the amygdalae and the insular cortex depends on stimulus reference (as described below). The authors further conclude that brain structures implicated in affective and self-related processing might be crucial prerequisites for a subjective experience of emotions. 
Reading unpleasant words increased activity in the amygdala and the insular cortex regardless of self, other or no reference (Figure 6 and 7). This confirms the role of these two structures in processing negatively valenced stimuli (negative affective information processing). Interestingly, positively valenced nouns preferentially increased amygdala and insular cortex activity in case of self-reference compared to other or no reference (Figure 6 and 7). In addition, pleasant nouns were rated more pleasant in case of self-reference, which mirrors underlying neurophysiology on the behavioural level.

Taken together, the above-mentioned studies highlight that "emotion"-related brain activity begins at the subcortical level (section 3.), and that self-reference seems to play a specific role for at least some of emotion-related information (section 4.).

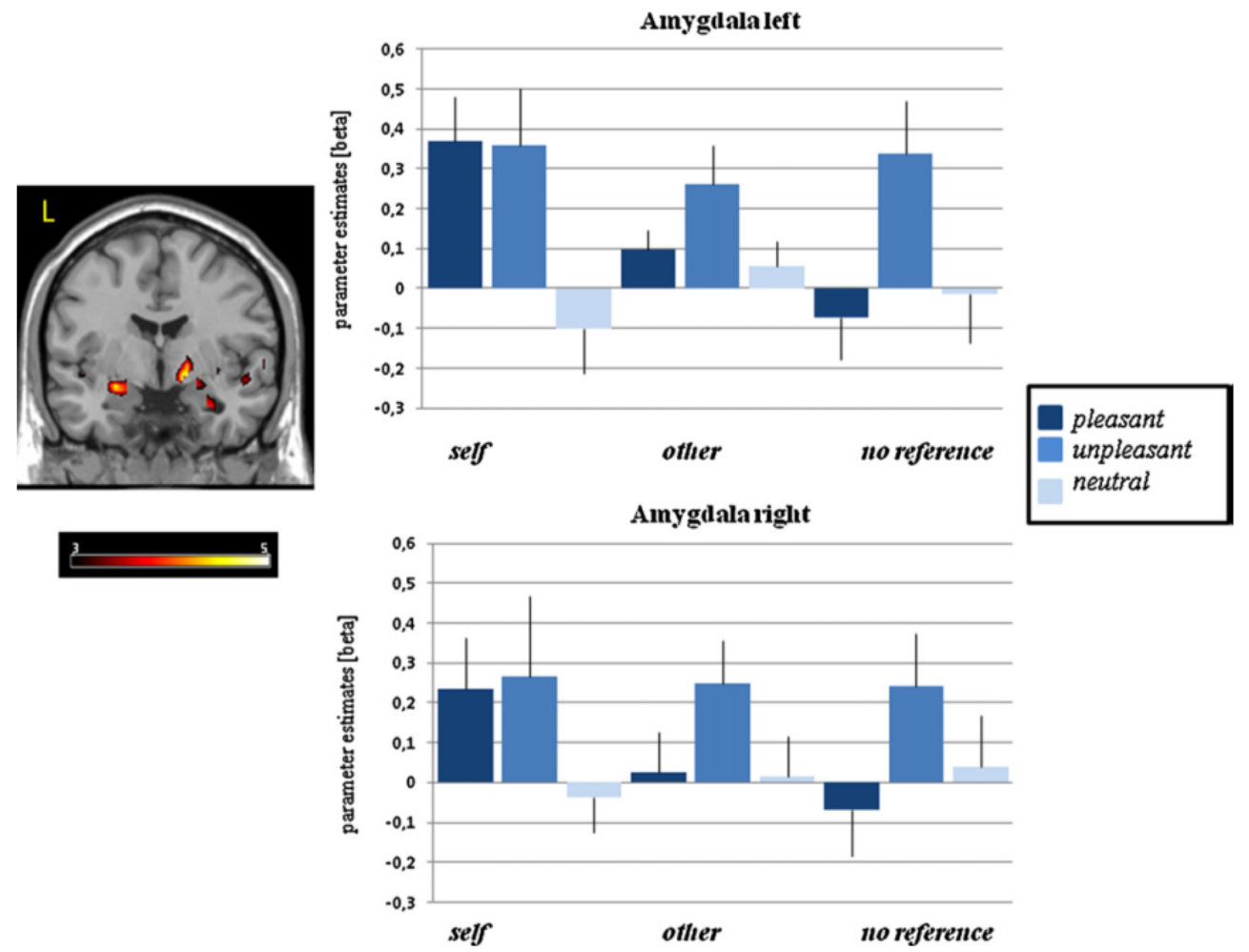

Figure 6. Right panel: Mean signal changes (mean and standard errors) in the left and right amygdala during processing of emotional and neutral self-related, other-related and unreferenced nouns. The picture on the left (left panel) displays significant changes in the amygdala during processing of selfrelated emotional nouns, $\mathrm{p}<0.005$. Color bars represent T-values. (adapted from Herbert et al., 2011b with permission). 

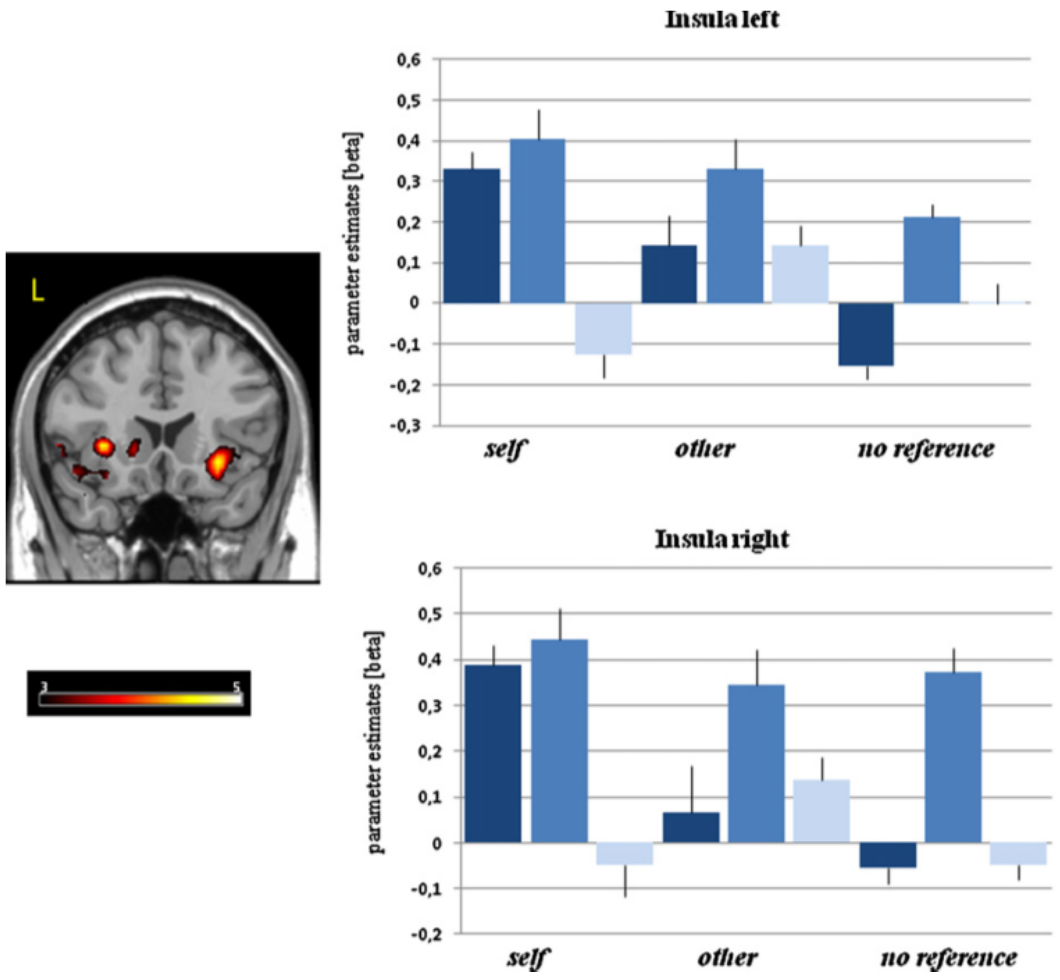

Figure 7. Right panel: Mean signal changes (mean and standard errors) in the left and right insula during processing of emotional and neutral self-related, other-related and unreferenced nouns. The picture on the left (left panel) displays significant changes in the insula during processing of self-related emotional nouns, $\mathrm{p}<0.005$. Color bars represent T-values. (adapted from Herbert et al. (2011b) with permission).

\section{Problems and inconsistencies}

Because of the many levels of control of all emotion-related processes within the neuroaxis, scholars from different disciplines discussing emotion issues easily misinterpret what others say. Some feel that emotions arise from higher brain regions where cognitions and affects intermingle; some think that discussions of emotions in animals must be constrained to the behavioral level only (LeDoux, 1996, 2012); yet others think that emotions are epiphenomena and can have no causal influence on the control of behavior. The view of too many behavioral neuroscientists - that there is no possibility of a sound science of experienced affective processing (feeling) - avoid the most robust evidence against their beliefs: Almost everywhere in the brain emotional behavior can be induced with DBS and can serve as "rewards" and "punishments" in the control of behavior. If behaviorist skeptics wish to have it their way, their responsibility is to list the diverse "rewards" and "punishments" which control human behavior that are not experienced in our species. In our experience, none have yet to be put forward. And from this vantage, experienced 
affective processing (feeling) has to be defined as much in neural terms as in psychological/verbal ones - for the confusion exists primarily at the later level.

If one is after a definition of emotion it turns out that almost as many definitions exist as textbooks are available. Just as some scholars would call the engine of a car "car", while others would call driving a hunk of sculpted metal a "car" without realizing that the engine is essential for driving, but it actually is a separate thing deserving its own label. Additionally, many original articles in emotion research report about emotions elicited in observers after presentations of facial expressions. Does a facial expression really elicit the same emotion-related activity in the observer's brain as the one that caused the facial expression in the person the face belongs to? It seems that the answer is, no it doesn't (see Sabatinelli et al., 2011; Britton et al., 2005, 2006). Looking at 157 neuroimaging studies, Sabatinelli found that only the amygdala demonstrated consistent activity independent from stimulus category (face or scene). Ideally, the science community needs a more specific definition of emotion in order to make consistent progress and to promote research communication within and across disciplines.

In the frame of this chapter we tried to consistently use the terms "affective information" and "emotion". In the original articles these terms were used interchangeably and even mixed with further terms including cognitive aspects. As mentioned in the introduction, we want to generate awareness about the inconsistent and broad use in particular of the term emotion. Feeling the urge to do something about this is not new. We are not the first to address this issue and we are certainly not alone. In 1981, Kleinginna and Kleinginna created awareness about the wide variety of approaches to labeling emotion by providing a list of no less than 92 emotion definitions. They classified these definitions into 11 categories depending on their theoretical basis. Some of these categories are based on subjective aspects of emotion (affective and cognitive), others on external stimuli, or on physiological mechanisms. Some are based on expressive behavior, on functional consequences or on disruptive or adaptive effects. Others are based on the multi-aspect nature of emotional phenomena and some distinguish emotion from other processes or emphasize the overlap between emotion and motivation. Finally, the authors found some statements that question the usefulness of the entire "emotion" concept. No doubt, the variety is big. Kleinginna and Kleinginna also stated that more recent definitions are as inconsistent as the earlier ones. This reflects the state of research up to the year 1981.

In 1995, LeDoux emphasized in the abstract of a paper entitled "Emotion: Clues from the Brain" that scientists at that time have not been able to reach a consensus about what emotion is and what place emotion should have in a theory of mind and behavior. Some emotions were considered more basic than others. To what extent do emotional responses contribute to emotional experiences? What can we say about interactions between cognition and emotion and finally how important are conscious versus unconscious processes in emotion research. A few years later, Cabanac (2002) mentioned that there is still no consensus in the literature on a definition of emotion and even today, a decade later, not much progress has been made. Perhaps, due to the appealing possibilities of neuroimaging methods researchers were spoiled by eye-catching colored brain images that looked nice 
and were easy to sell, but after all they neglected to make progress in terms of identifying a better concept of emotion and providing a more specific evidence-based definition. Fed by multi-used terms for brain functions that occur from sensory input to actual behavior, ongoing research is obviously not focused on clarifying terminological issues around "emotion", so semantic confusions prevail. To date, we still lack a useful definition and a distinct and common terminology of related functions.

However, we would suggest that a nested hierarchical view of both brain and mind may go a long way toward clarity, in that discourse in the area, especially as related to human emotions, has been primarily at what we call the tertiary process level. If we consider that primary-process analysis, best pursued in animal models (Panksepp, 1998, 2011a,b), provides the most robust evidence for cross-mammalian genetically-ingrained infrastructures for emotionality, not only in terms of behavior but raw phenomenally significant affective experiences, we may need to cultivate new ways to discuss the full complexity of the brain and mind (Solms \& Panksepp, 2012). The single fact that highlights the affective phenomenology that arises from these ancient circuits is the simple fact that wherever in the brain investigators have evoked, with deep brain stimulation, coherent instinctual expressions of emotional behaviors, those central states always serve as rewards and punishments in the control of simple learned approach and escape behaviors (even in the absence of neocortex). Since all major "rewards" and "punishments" that control human behavior are also experienced affectively, we have no good reasons to conclude, other than pervasive biases promoted by long-standing beliefs, that the primitive subcortical foundations of emotional integrations that are experienced as affects cannot be cognitively interpreted by our brains. We think they can, but this requires a new marriage between animal and human research, where one recognizes how powerfully cognitive aspects of mind can influence affective functions and vice versa. How those findings relate to the abundant correlative data from human brain imaging is an open issue (Lindquist et al., 2012), but one needs to conceptualize and evaluate the cognitive and affective issues independently for the reciprocal interactions can be huge (Liotti and Panksepp, 2004; Northoff, et al., 2009).

An informative review paper by Phan et al. (2002) summarizing 55 Neuroimaging studies (Positron Emission Tomography (PET) and functional Magnet Resonance Imaging (fMRI)) nicely demonstrates that authors label "emotion" almost anything that happens in the brain without discriminating between affective processing, actual subjective experiences and other aspects (Figure 8). Phan et al. stated as one of the inclusion criteria that only studies about "higher-order mental processes of emotion" were entered in their analysis, while excluding lower-order processes including "sensory or motor processes, such as gustatory/olfactory or pain induction". This distinction together with the wide distribution of relevant brain areas highlights that emotion-related brain processing does have many aspects, but many traditional distinctions do not really help to better understand them nor do they help to better define emotion. In fact, many facets of the current debate about the nature of emotions continue to contribute to the many confusions that have long characterized this field (for a many examples, and suggested solutions, see Lindquist, et al., 2012). 
Neuroimaging Helps to Clarify Brain Affective Processing Without Necessarily Clarifying Emotions 109

A

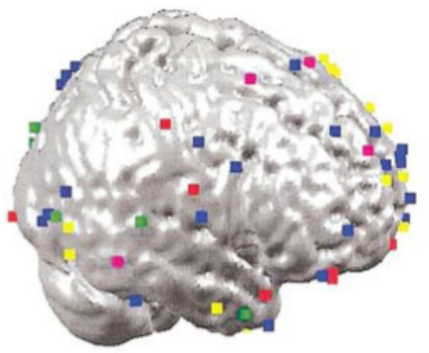

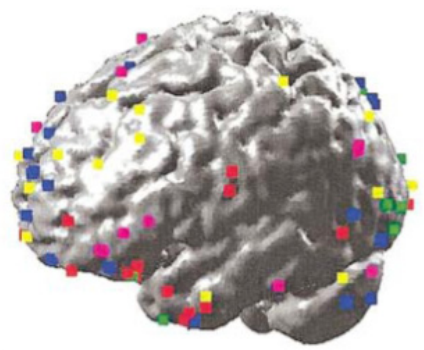

\begin{tabular}{|l}
$\square$ Happiness \\
Sadness \\
Disgust \\
Fear \\
Anger
\end{tabular}
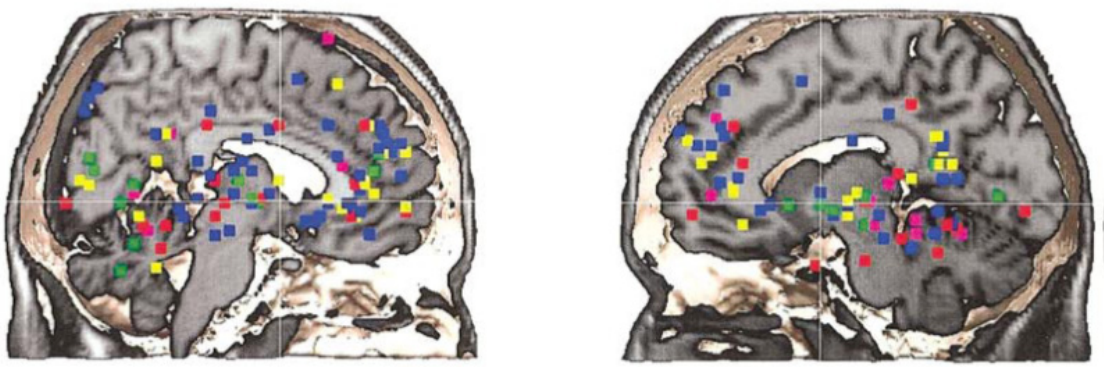

B
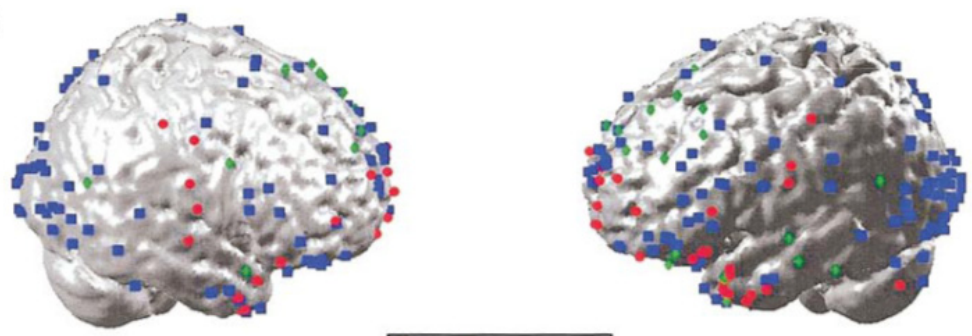

Visual

Recall
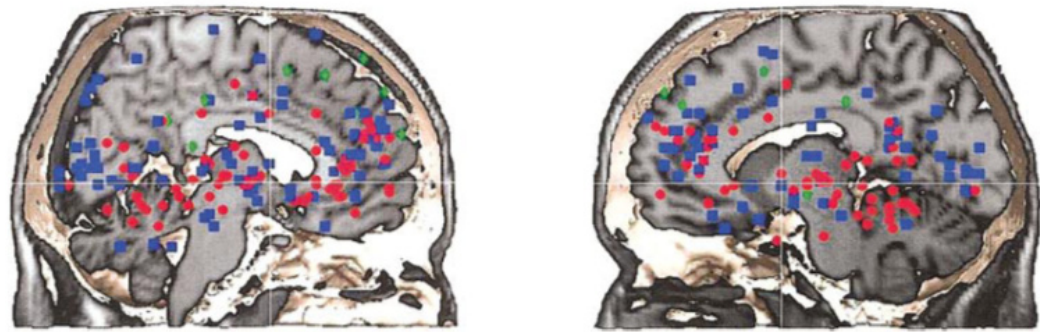

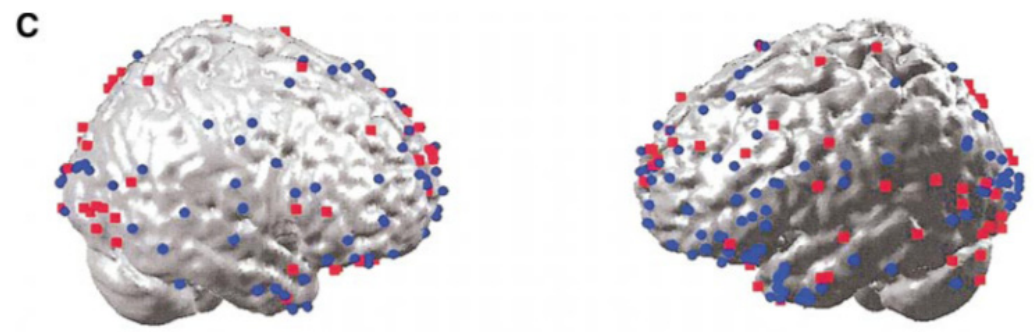

\section{Emotion + cognition \\ Emotion alone}
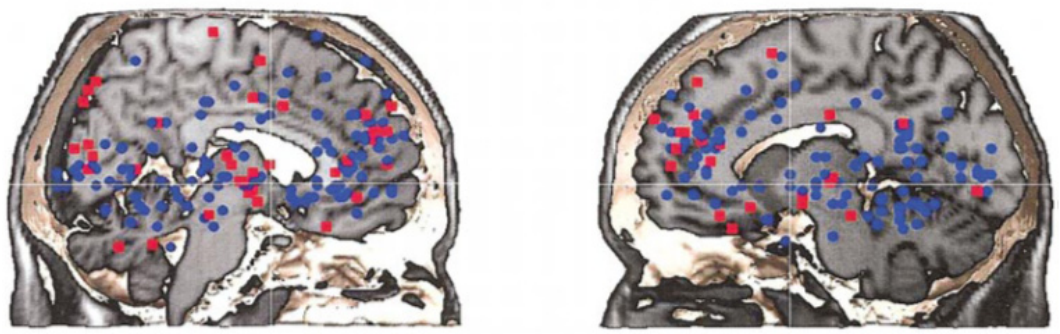

Figure 8. Phan et al. (2002) study: Overlay of neuroimaging findings from emotion studies showing emotion-relevant brain areas. (A) Activation foci: Individual emotion. (B) Activation foci: Induction method. (C) Activation foci: Cognitive demand. Adapted from Phan et al. (2002) with permission.

Too many chefs spoil the soup and too many scholars from different disciplines spoil the definition of emotion. For cognition, the sibling of affective information processing (both system are meant to support behaviour adaptation), you can say that it basically means "thinking", but breaking emotion down to a single term seems to be a rather difficult challenge. The reason for that is because emotion is the output and not the processing. If you place affective information processing on the level of cognitive information processing you could say that affective processing means "evaluating". In this chapter, it is suggested that the term emotion be reserved for only the behavioral aspects of it. After all, the Latin word emotion means to move out. It is about movement and movement equals behavior (in terms of neurobiology). The alternative is that emotion be simply seen as a class-identifier-an umbrella concept - that includes all the major aspects that are traditionally subsumed by this concept, namely the behavioral, autonomic-physiological, neural, cognitive and affective attributes. The reason we suggest the more minimalist solution is that the broader concept has led to so much trouble already, and at this stage of history we find many people talking past each other (as is well summarized in Lindquist, et al., 2012).

This would allow the experiential issues to be discussed more cogently without the excess historical baggage that now clutters the field and continually impedes clear discourse. If we were to reserve the term "emotion" to the visually evident behavioral displays, and discussed affective states independently at several distinct levels-e.g., the primal affective- 
experiential level, the secondary-process learning level, and the tertiary-process thoughtful cognitive level, we may be able to disentangle the conceptual Gordian Knot that is the major current historical dilemma of the field. If we do this, we can envision the study of affect related cognitive information-processing to highlight many of the unique properties of the affective life in humans, and the many ancestral affective value schemata apparently shared homologously in all mammals (maybe even all vertebrates) to provide a level of fundamental psychological analysis that is best pursued constitutionally and causally in animal models of emotionality. This would still leave the many important autonomicvisceral arousals that accompany emotions as a field that can be equally illuminated by human and animal research. Such an approach would also allow for several different affects and perhaps emotions to occur simultaneously as a result of one set of external stimuli, affective stimuli.

\section{The proposed Emotion-Model}

The proposed Emotion-Model (figure 9) follows the idea that simpler schemes are better than complex ones, especially in the early phases of investigation, where we still are despite thousands of published papers. Our model gives the ambiguous term "emotion" a distinct place (and meaning) rather than using it interchangeably for various different processes that should all have their own separate labels. We were inspired by numerous neuroimaging studies (e.g. especially Damasio et al., 2000), which mainly demonstrated the engagement of subcortical structures in processing of affectively experienced states. We follow Panksepp's idea that "emotions" have their behavioural-action roots deep in the brain, and they also generate raw feelings, and label the roots "affective processing". We also accept that the lower levels of processing are strongly influenced by higher brain functions which are most effectively studied in humans (e.g, Liotti \& Panksepp, 2004; Northoff, et al., 2006; Lindquist, et al., 2012). Because the foundational structures for emotionality are old in evolutionary terms and because it is often claimed that conscious experiences rely on cortical structures, we believe that "emotions", as behaviors, all have an unconscious origin, while at the same time, through some kind of poorly understood emergent "field dynamics" those emotionrelated systems provide a foundation for the origin of raw affective feelings. Their origin is ultimately rooted in primal affective action routines as well as higher affective information processing. Still, the first level of affective information processing, as reflected in basic mechanisms of learning and memory is unconscious by nature. It does not always promote emotional displays, while still processing stimulus-related life supportive or detrimental aspects. In other words, the intermediate secondary-process level that lies between our lower and higher psychological functions may help parse action patterns in space and time, and thereby provide more discrete pieces of information for higher brain processing. Many human emotions are possible consequences of such unconscious processing of affective states into more discrete components that are discrete motor expressions in one way or another. They occur in humans and certainly in various other nonhuman mammals. It may even be that such affective processing without creating any new emotional reactions occurs in some if not all lower vertebrates. In humans, such intermediate levels of affective 
processing provide enormous amounts of new information that is grist for thought, as well as the generation of enormous affective and emotional nuances.

The behavioural aspects of emotions (or expressive aspects) were highlighted by several authors. Even Darwin focussed on the expressive nature of an emotion rather than anything else, although he never denied the affective power of emotions. We now know more about the underlying processes that lead to an emotion, and what we can derive from the evidence is that not all affective processes (especially sensory and homeostatic ones) necessarily lead to emotion responses, although they guide our behaviour on the basis of evaluating life supportive or detrimental aspects of information representing the outer world. If these processes do not lead to emotions and also not to feelings, which are experienced affective processes they remain non-conscious and are thus a type of incidental encoding, which we call here incidental affective encoding.

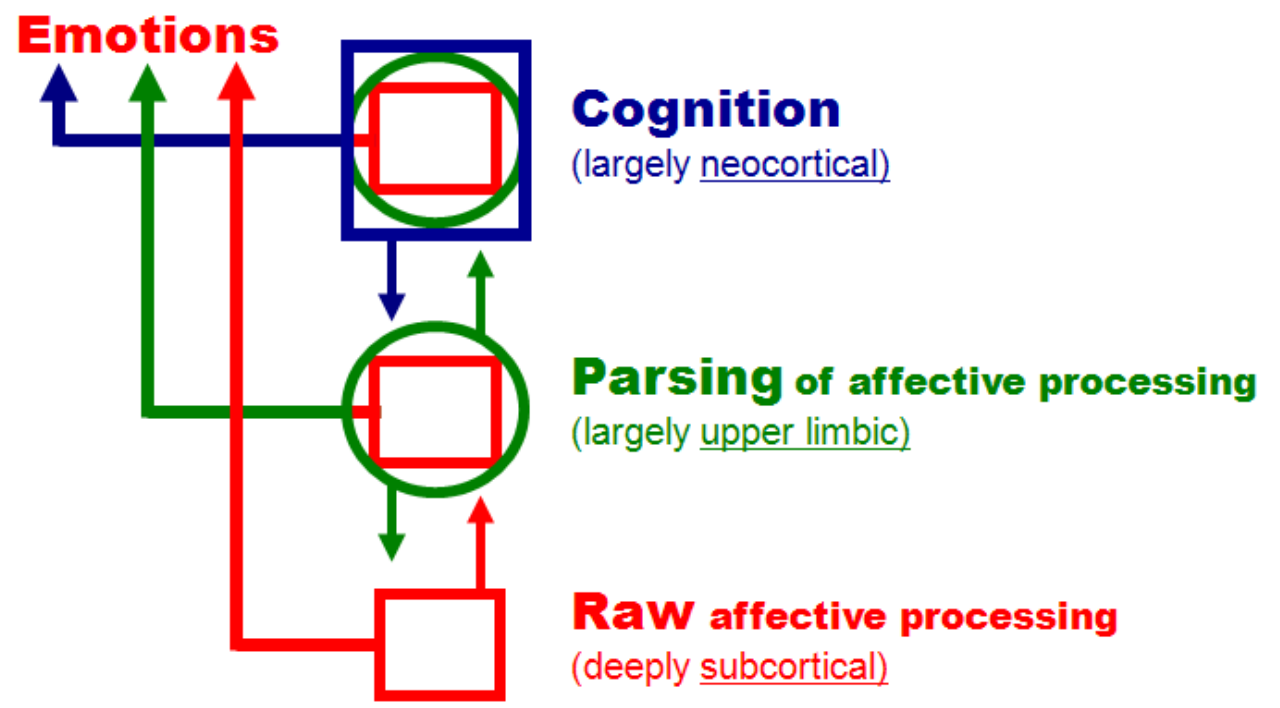

Figure 9. The proposed emotion model. Emotions are consequences of raw affective processing. They can be modified by information of higher order processes. Thus, raw affective information is able to elicit more than just one emotion, while these multiple emotions can even be differently valenced. However, affective processing also occurs in the absence of emotion generation. Finally, emotions are principally independent from cognition. Adapted from Solms and Panksepp (2012).

To summarise the proposed Emotion-Model, an emotion is always caused by implicit affective information processing. Even emotional displays in humans are thus the result of affective information processing. Affective information processing in humans typically evaluates the world outside and only sometimes, when certain intrinsic types of withinbrain action readiness schema are aroused, create both emotional responses and emotional feelings. It may well be that the crucial factor for creating an emotion and/or a feeling as a result of affective processing is deep "self reference" as it may occur in brain regions as deep as the PAG (Panksepp, 1998) as well as higher order "self reference" as it may occur in the 
insular cortex (Walla et al., 2007; Herbert et al., 2011b) and various medial cortical zones (Northoff, et al., 2006).

"Emotion" research is a field with wide implications. Basic researchers, clinicians, product developers and also advertisers and marketers - they all deal with emotion, yet they don't seem to bother too much about the fact that one term - emotion - is in use to explain a whole complex chain of brain processes and perhaps different functions and/or subfunctions. The present multi-tiered evolutionary model that we propose here may help to guide scientific inquiries and explain scientific findings in more coherent, and hopefully understandable ways. It must be noted that most other animals do not have such massive higher reflections of cognitively experienced affective processing, so in them instinctual emotional behaviours may veridically reflect corresponding affective states (Panksepp, 1998, 2011a,b). Thus, animal research may help illuminate the subcortical emotional and affective infrastructures of human minds that are comparatively inaccessible through human research. An example of how this may promote understanding of human emotional responses is currently well-represented in the emerging field of neuroeconomics (Knutson \& Greer, 2008) as well as consumer neuroscience, where intuition, or in other words the well known "gut-feelings", are in discussion as implicit affective processes, perhaps merged with non-conscious cognitions. For instance, startle reflex modulation is considered to be a methodological approach that allows to quantify affective information processing in the human brain (e.g. Walla et al., 2010; Walla et al., 2011; Geiser et al., 2011; Grahl et al., 2012).

Our final statement relates to the idea that affective information processing is adaptive and supports the fitness of an organism as usually seen from an evolutionary perspective (see Gross, J.J., 1999). We believe that at least in humans this is not always the case, since the primary-process subcortical networks for affective processing emerged long before the human neocortex, which permits self-reflective thoughtfulness about ones affective feelings. It may well be that affective information processing and emotions do play an adaptive role, but obviously they can be life threatening as in cases of severe depression. Depressions are assumed to have affective origins, closely related to various primary-process affective systems (Coenen, et al., 2011; Panksepp \& Watt, 2011). They can cause enormous life problems if not adequately dealt with. Perhaps, this issue often does not relate to affective processing alone, but often occurs as a result of affective processing associated with "internal information" such as information stored in episodic memory, which can lead to ruminative dwelling on one's emotional problems.

If so, psychotherapy without concurrent use of optimal medications may not be as effective as with the use of affectively appropriate medications, which can now be developed through the use of animal models that respect and study the emotion related feelings of other animals (e.g., Burgdorf, et al., 2011; Panksepp, 2012). Indeed, if the "primary affective processing" substrates have been sensitized (e.g., elevated responsivity of the FEAR system in chronic anxiety and PTSD), then pharmacological somatic approaches may need to be implemented promptly to alleviate distress. Since such circuit changes are not easily monitored in individual clients, it may be judicious to assume such changes may have transpired in the underlying primal emotional substrates, and evaluate whether modest 
doses of appropriate medications can facilitate psychotherapeutic interventions. The Emotion-Model as proposed here does not yet include a full discussion of optimal psychotherapeutic aspects. However, the stratified emotion model we have been working with (Figure 9) highlights how higher and lower aspects of affective information processing may need to be envisioned for the generation of a comprehensive model (for further development of this nested-hierarchical model, see Northoff, et al., 2011; Panksepp, 2011). We simply offer this chapter as an introductory start. The model will be further developed and future investigations will show whether the suggested hierarchical structure can accommodate the fuller complexity of human affective information processing, its accompanying affective states and accompanying emotional responses.

\section{Author details}

Peter Walla

School of Psychology, University of Newcastle,

Center for Translational Neuroscience and Mental Health, Australia

Jaak Panksepp

Department of VCAPP, College of Veterinary Medicine, Washington State University, USA

\section{References}

Alcaro, A., Huber, R., \& Panksepp, J. (2007). Behavioral functions of the mesolimbic dopaminergic system: An affective neuroethological perspective. Brain Research Reviews, 56, 283-321.

Alcaro, A. \& Panksepp, J. (2011). The SEEKING mind: primal neuro-affectie substrates for appetitive incentive states and their pathological dynamics in addictions and depression. Neuroscience \& Biobehavioral reviews, 35(9): 1805-20.

Adolphs, R., Tranel, D., Damasio, H., and Damasio, A. R. 1995. Fear and the human amygdala. J. Neurosci. 15: 5879-5891.

Barrett, Mesquita, et al. (2011). "Context in Emotion Perception." Current Directions in Psychological Science 20(5): 286-290.

Bechara, A., Tranel, D., Damasio, H., Adolphs, R., Rockland, C., and Damasio, A. R. (1995). Double dissociation of conditioning and declarative knowledge relative to the amygdala and hippocampus in humans. Science 269: 1115-1118

Berlin, H.A. (2011). The neural basis of the dynamic unconscious (with commentaries). Neuropsychoanalysis, 13, in press.

Berridge, K.C., Winkielman, P. (2003). What is an unconscious emotion? (The case for unconscious "liking"). Cognition and Emotion, 17(2): 181-211.

Britton, J.C., Phan, K.L., Taylor, S.F., Welsh, R.C., Berridge, K.C., and Liberzon, I. (2005). Neural correlates of social and non-social emotions: an fMRI study. Neuroimage, 31(1):397-409. 
Britton, J.C., Taylor, S.F., Berridge, K.C., Mikels, J.A., Liberzon, I. (2006). Differential subjective and psychophysiological responses to socially and nonsocially generated emotional stimuli. Emotion 6(1):150-155.

Burgdorf, J., Panksepp, J., \& Moskal, J. R. (2011). Frequency-modulated 50kHz ultrasonic vocalizations: a tool for uncovering the molecular substrates of positive affect. Neurosci Biobehav Rev, 35(9): 1831-1836.

Cabanac, M. (2002). What is emotion? Behavioural Processes 60: 69-83.

Calder, A. J., Young, A. W., Rowland, D., Perrett, D. I., Hodges, J. R., and Etcoff, N. L. (1996). Facial emotion recognition after bilateral amygdala damage: Differentially severe impairment of fear. Cogn. Neuropsychol. 13: 699-745.

Coenen, V.A., Schlaepfer, T.E., Maedler, B., Panksepp, J. (2011). Cross-species affective functions of the medial forebrain bundle-implications for the treatment of affective pain and depression in humans. Neuroscience \& Biobehavioral Reviews, 35(9): 1971-81.

Craig, A.D., How do you feel--now? The anterior insula and human awareness. Nat. Rev. Neurosci. 2009, 10, 59-70.

Damasio, A.R., Grabowski, T.j., Bechara, A., Damasio, H., Ponto, L.L., Parvizi, J., et al. (2000). Subcortical and cortical brain activity during the feeling of self-generated emotions. Nature Neuroscience, 3: 1049-1056.

Damasio, A., Damasio, H. \& Tranel, D. (2012). Persistence of feelings and sentience after bilateral damage of the insula. Cerebral Cortex, in press. doi:10.1093/cercor/bhs077

Darwin, C. R. 1859. On the origin of species by means of natural selection, or the reservation of favoured races in the struggle for life. London: John Murray. [1st edition].

Ekman, P., Friesen, W. V., O'Sullivan, M., Chan, A., Diacoyanni-Tarlatzis, I., Heider, K., Krause, R., LeCompte, W.A., Pitcairn, T., Ricci-Bitti, P. E., Scherer, K.R., Tomita, M., \& Tzavaras, A. (1987). Universals and cultural differences in the judgments of facial expressions of emotion. Journal of Personality and Social Psychology, 53(4), 712-717.

Garrett, A.S., Maddoc, R.J. (2006). Separating subjective emotion from the perception of emotion-inducing stimuli: an fMRI study. Neuroimage, 33: 263-274.

Geiser, M., Walla, P. (2011). Objective measures of emotion during virtual walks through urban environments. Applied Sciences, 1: 1-11.

Grahl, A., Greiner, U., \& Walla, P. (2012). Bottle shape elicits gender-specific emotion: a startle reflex modulation study. Psychology, 3(7): 548-554.

Gross, J.J. (1999). Emotion regulation: Past, Present, Future. Cognition and Emotion, 13(5): 551-573.

Halgren, E., Walter, R. D., Cherlow, D. G., and Crandall, P. H. (1978). Mental phenomena evoked by electrical stimulation of the human hippocampal formation and amygdala. Brain 101: 83-117.

Hamm, A.O., Weike, A.I., Schupp, H.T., Treig, T., Dressel, A., and Kessler, C. (2003). Affective blindsight: intact fear conditioning to a visual cue in a cortically blind patient. Brain, 126: 267-275. 
Herbert, C., Herbert, B.M., \& Pauli, P. (2011b). Emotional self-reference: Brain structures involved in the processing of words describing one's own emotions. Neuropsychologia, 49, 2947-2956.

Herbert, C., Herbert, B.M., Ethofer, T., \& Pauli, P. (2011a) His or mine? The time course of self-other discrimination in emotion processing. Social Neuroscience, 6, 277-288.

Izard, C. E. (1971). The face of emotion (Vol. 23): Appleton-Century-Crofts New York.

Kaplan-Solms, K. and Solms, M. (2000). Clinical Studies in Neuro-Psychoanalysis: introduction to a depth neuropsychology. London: Karnac Books.

Kleinginna, P.R.Jr., and Kleinginna, A.M. (1981). A Categorized List of Emotion Definitions, with Suggestions for a Consensual Definition. Motivation and Emotion, 5(4: 345-379.

Knutson, B., \& Greer SM. (2008). Anticipatory affect: neural correlates and consequences for choice. Philosophical Transactions of the Royal Society, London B Biological Sciences. 363: 3771-3786.

Ikemoto, S. \& Panksepp, J. (1999). The role of the nucleus accumbens dopamine in motivated behavior: a unifying interpretation with special reference to reward-seeking. Brain Research. Brain Research Review, 31(1): 6-41.

LeDoux, J.E. (1993). Emotional memory systems in the brain. Behavi. Brain Res. 58: 69-79.

LeDoux, J.E. (1995). Emotion: Clues from the Brain. Annu. Rev. Psycho, 46:209-235.

LeDoux, J. (1996). The emotional brain. New York: Simon \& Schuster.

LeDoux, J. (2012). Rethinking the emotional brain. Neuron, 73: 653-676.

Lindquist, K.A., Wager, T.D., Kober, H., Bliss-Moreau, E., and Barrett, L.F. (2012). The brain basis of emotion: A meta-analytic review. Behavioral and Brain Sciences, 35: 121-202.

Lindquist, K. A., Wager, T. D., Bliss-Moreau, E., Kober, H., \& Barrett, L. F. (2012). What are emotions and how are they created in the brain? Behavioral and Brain Sciences, 35: 175184. response to comments.

Liotti, M., \& Panksepp, J. (2004). On the neural nature of human emotions and implications for biological psychiatry. In Panksepp J (ed) Textbook of Biological Psychiatry, pp. 33-74. Wiley, New York.

Mauss, I.B., and Robinson, M.D. (2009). Measures of emotion: a review. Cognition and Emotion, 23(2): 209-237.

Morris, J.S., Öhman, A., and Dolan, R.J. (1999). A subcortical pathway to the right amygdala mediating "unseen" fear. Proc. Natl. Acad. Sci., 96(4): 1680-1685.

Narumoto, J., Okada, T., Sadato, Fukui, K., Yonekura, Y. (2001). Attention to emotion modulates fMRI activity in human right superior temporal sulcus. Cognitive Brain Research, 12: 225-231.

Northoff, G., Henzel, A., de Greck, M., Bermpohl, F., Dobrowolny, H., \& Panksepp, J. (2006). Self-referential processing in our brain-A meta-analysis of imaging studies of the self. Neuroimage, 31, 440-457.

Northoff, G., Schneider, F., Rotte, M., Matthiae, C. Tempelmann, C., Wiebking, C., Bermpohl, F., Heinzel, A., Danos, P., Heinze, H.J., Bogerts, B., Walter, M., \& Panksepp, 
J. (2009). Differential parametric modulation of self-relatedness and emotions in different brain regions. Human Brain Mapping, 30, 369-382.

Northoff, G., Wiebking, C., Feinberg, T, and Panksepp, J. (2011). The'resting-state hypothesis' of major depressive disorder - a translational subcortical-cortical framework for a system disorder. Neurosci. Biobehav. Rev. DOI: 10.1016/j.neubiorev.2010.12.007

Öhman A. 2002. Automaticity and the amygdala: nonconscious responses to emotional faces. Curr.Dir. Psychol. Sci. 11:62-66

Panksepp, J. (1992). A critical role for affective neuroscience in resolving what is basic about basic emotions. Psychological Review, 99, 554-60.

Panksepp J. (1998). Affective Neuroscience: The Foundations of Human and Animal Emotions (Series in Affective Science). Oxford University Press, New York, New York.

Panksepp, J. (2005). On the embodied neural nature of core emotional affects. Journal of Consciousness Studies, 12, 158-184.

Panksepp, J. (2011a). Cross-species affective neuroscience decoding of the primal affective experiences of humans and related animals. PloSOne, 6(9).e21236.

Panksepp, J. (2011b). The basic emotional circuits of mammalian brains: do animals have affective lives?. Neurosci Biobehav Rev, 35(9): 1791-804.

Panksepp, J. (2012). The vicissitudes of preclinical psychiatric research: justified abandonment by big pharma? Future Neurology, 7: 1-3.

Panksepp, J. \& Biven, L. (2012). Archaeology of Mind: The Neuroevolutionary Origins of Human Emotions. New York: Norton.

Panksepp, J. \& Moskal, J. (2008). Dopamine and SEEKING: subcortical "reward" systems and appetitive urges. In: Elliot, A. (Ed.), Handbook of Approach and Avoidance Motivation. Mahwah, NJ: Lawrence Erlbaum Associates, pp. 67-87.

Panksepp, J., \& Watt, D. (2011). What is basic about basic emotions? Lasting lessons from affective neuroscience. Emotion Review, 3, 387-396.

Phan, K.L., Wager, T., Taylor, S.F., and Liberzon, I. (2002). Functional Neuroanatomy of Emotion: A meta-Analysis of emotion activation studies in PET ans fMRI. Neuroimage, 16: 331-348.

Sabatinelli, D., Fortune, E.E., Li, O., Siddiqui, A., Krafft, C., Oliver, W.T.,Beck, S., Jeffries, J. (2011). Emotional perception: Meta-analyses of face and natural scene processing. NeuroImage, 54(3): 2524-2533.

Solms, M. \& Panksepp, J. (2012). The 'Id' Knows More than the 'Ego' Admits: Neuropsychoanalytic and Primal Consciousness Perspectives on the Interface between Affective and Cognitive Neuroscience. Brain Science, 2, 147-175. doi:10.3390/brainsci2020147

Tracy, J.L. and Randles, D. (2011). Four models of basic emotions: a review of Ekman and Cordaro, Izard, Levenson, and Panksepp and Watt. Emotion Review, 3(4): 397-405.

Tracy, J. L., \& Robins, R. W. (2008). The automaticity of emotion recognition. Emotion, 8(1), 81-95. 
Walla, P. (2011). Non-Conscious Brain Processes Revealed by Magnetoencephalography (MEG), Magnetoencephalography, Elizabeth W. Pang (Ed.), ISBN: 978-953-307-255-5, InTech, Available from:

http://www.intechopen.com/books/magnetoencephalography/non-conscious-brainprocesses-revealed-by-magnetoencephalography-meg-

Walla, P., Brenner, G., and Koller, M. (2011). Objective measures of emotion related to brand attitude: A new way to quantify emotion-related aspects relevant to marketing. PlosOne, 6(11): e26782. doi:10.1371/journal.pone.0026782.

Walla, P., and Deecke, L. (2010). Odours Influence Visually Induced Emotion: Behavior and Neuroimaging. Sensors, 10(9):8185-8197.

Walla, P., Richter, M., Färber, S., Leodolter, U., and Bauer, H. (2010). Food evoked changes in humans: Startle response modulation and event-related potentials (ERPs). Journal of Psychophysiology, 24(1): 25-32.

Walla, P. (2008). Olfaction and its dynamic influence on word and face processing. Progress in Neurobiology: Cross-modal integration. Progress in Neurobiology, 84: 192-209.

Walla, P., Duregger, C., Greiner, K., Thurner, S., and Ehrenberger, K. (2008). Multiple aspects related to self awareness and the awareness of others: an Electroencephalography (EEG) study. Journal of Neural Transmission, 115(7):983-92.

Walla, P., Greiner, K., Duregger, C., Deecke, L., and Thurner, S. (2007). Self awareness and the subconscious effect of personal pronouns on word encoding: a magnetoencephalographic (MEG) study. Neuropsychologia, 45: 796-809.

Zachar, P., \& Ellis, R. (Eds.). (2012). Emotional theories of Jaak Panksepp and Jim Russell. Amsterdam: John Benjamins.

Zajonc, R. B. (2000). Feeling and thinking: Closing the debate over the independence of affect. In J. P. Forgas (Ed.), Feeling and thinking: The role of affect in social cognition (pp. 31-58). New York: Cambridge University Press. 